\title{
LA GOBERNANZA DEL AGUA Y LA PARTICIPACIÓN CIUDADANA EN BOGOTÁ*
}

\section{Water governance and citizen participation in Bogotá}

\author{
Ricardo Motta Vargas** \\ Néstor Raúl Ramírez Moreno*** \\ Corporación Universitaria Republicana. Bogotá, D.C.
}

Recepción: 16 de marzo de 2016. Aceptación: 22 de julio de 2016.

DOI: http://dx.doi.org/10.21017/Rev.Repub.2016.v21.a16

\section{RESUMEN}

Introducción. Investigación que analiza el proceso social y jurídico que han desarrollado comunidades ambientalistas del Distrito Capital, logrando la participación, en la gobernanza del agua, para la gestión integral de sus fuentes hídricas. Método. Se ha utilizado una metodología de tipo documental descriptiva, a partir de la revisión de material proveniente de los estudios acerca de la participación ciudadana en Bogotá D.C. Resultados. Los Cabildos para la Gobernanza del Agua (CGA) tienen como sus antecedentes históricos, los movimientos sociales y cívicos que se originaron en los años noventa, en la defensa de sus territorios, deteriorados por la problemática ambiental, la expansión urbana, la invasión de la ronda de los ríos y el mal manejo de los desechos industriales y tóxicos. La política pública en Bogotá D.C.,

* Artículo resultado del proyecto de investigación: El derecho de participación en la gobernanza del agua en Bogotá, desarrollado por los grupos Derecho Económico y Estado - Derecho Público y financiado por la Corporación Universitaria Republicana.

** Investigador principal en el proyecto: El derecho de participación en la gobernanza del agua en Bogotá. Docente-investigador con títulos de Maestría en Educación, Universidad Libre y las Tunas - Cuba. Especialista en Derecho Administrativo del Colegio Mayor del Rosario, en Derecho Constitucional de la Universidad Nacional, y en Teoría Jurídica de la Universidad Libre. Abogado de la Universidad Externado de Colombia. Autor del libro Ordenamiento Territorial en el Quinquenio de Rafael Reyes y de otros ensayos jurídicos publicados en diversas revistas académicas. Docente investigador de la Corporación Universitaria Republicana. Correo electrónico: mottaricardo2003@yahoo.com

*** Investigador principal en el proyecto Intervención y manejo de cuencas hidrográficas en áreas urbanas. El caso del río Fucha en la ciudad de Bogotá D.C. Integrante del grupo de Derecho Económico y Estado de la Corporación Universitaria Republicana, reconocido por Colciencias. Profesor con estudios de Maestría en Análisis de Problemas Políticos, Económicos e Internacionales Contemporáneos, Licenciado en Ciencias Sociales, Diplomado en Derechos Humanos. Correo electrónico: nestoramirezmo@yahoo.es 
desarrollada a partir del 2012, de gobernanza del agua, ha fomentado la participación ciudadana con los CGA, los cuales han tenido incidencia en el manejo y gestión de los sistemas hídricos sobre los cuales están asentadas las comunidades. Conclusión. Las experiencias de algunos movimientos sociales, y la instalación y desarrollo de 43 CGA, no han sido suficientes en sus alcances, para un goce pleno en la gestión del recurso hídrico, lo que permite concluir que una buena gobernanza del agua se debe fundamentar principalmente en el liderazgo de las comunidades y en la búsqueda del consenso de todos los actores intervinientes, lo cual permitirá generar políticas públicas con justicia social en la gestión del agua.

Palabras clave: Movimientos sociales, gobernanza del agua, derecho de participación.

\begin{abstract}
Introduction. Research that analyzes the social and legal process that have developed environmental communities of the Capital District, achieving participation in water governance for integrated management of their hydric sources. Method. A descriptive documental methodology has been undertaken, through material originated from studies about citizen participation in Bogotá D.C. Results. The councils for water management CGA have as historical background, social and civil movements that were originated in the 90s, for the defense of their territories, deteriorated by environmental problems, urban expansion, the invasion around rivers and the mismanagement of industrial and toxic waste public policy in Bogota D.C. The public policy of water governance developed in Bogota D.C. since 2012, has encouraged citizen's participation with the CGA, having had an impact on the handling and management of water system on which the communities are settled. Conclusion. The experiences of some social movements, and the installation and development of 43 CGA, haven't been enough in their scopes, for a full enjoyment in the management of water resources, which allows to conclude that a good governance of water should be mainly based on the community leadership and in the search of the consensus of all involved actors, so as to generate public polices with social justice in water management.
\end{abstract}

Key words: Social movements, water management, participation rights.

\title{
1. INTRODUCCIÓN
}

A partir del II Foro Mundial del Agua celebrado en la ciudad de La Haya (Holanda), en el año 2000, se puso en debate para el mundo el tema de la 
gobernanza del agua (VI Foro Mundial del Agua, 2012, p. 10). Se tienen como importantes antecedentes: la recuperación del río Sena en la ciudad de París, en 1990, entre el Estado francés y la comunidad como gobernante de este recurso hídrico; y la experiencia de la ciudad de Nueva York, también en los años noventa, en donde los usuarios, las comunidades locales de los condados de la ciudad, pagaban por la gestión y mantenimiento de cuencas a particulares; este último fue un interesante programa de recuperación y conservación de los ecosistemas, después de varios acuerdos entre la comunidad y la administración local.

El IV Foro Mundial del Agua, realizado en la ciudad de México en 2006, planteó que

la «buena gobernanza del agua» debía incluir «la acción coordinada a diferentes niveles (principio de subsidiaridad), la transparencia y la participación pública de todos los sectores (ciudadanía activa), incluyendo las mujeres y niños». La gobernanza se perfila así, dentro del ámbito ambiental, y especialmente referido a los recursos hídricos, como el enfoque teórico más idóneo para hablar de un gobierno de los recursos efectivamente participativo, dentro del esquema de la Gestión Integrada de los Recursos Hídricos (GIRH), es decir, con esquemas descentralizados de toma de decisiones. (VI Foro Mundial del Agua, 2012, p. 11).

A partir de este momento muchos Estados iniciaron políticas públicas dirigidas hacia la promoción de procesos para la gobernanza del agua, vinculando como actores sociales a las comunidades relacionadas con la gestión del agua y la recuperación de cuencas hidrográficas.

En los posteriores foros del agua se han estado discutiendo diferentes enfoques de la gobernanza del agua: un primer enfoque considera el agua como una necesidad valorada en dinero y no como un derecho de todos; un segundo enfoque considera el agua como un bien público para todos y la gobernanza y gestión integral del agua con justicia social.

El enfoque de gobernanza del agua con justicia social promovió en Colombia movimientos en defensa del agua como derecho humano, el derecho a la participación ciudadana en la gestión de los ecosistemas hídricos, y la presión social para la descontaminación de fuentes hídricas.

Desde finales de los años noventa se han venido conformando en la ciudad de Bogotá D. C., sin el apoyo estatal, movimientos sociales para la recuperación de humedales, quebradas y algunas cuencas hidrográficas y para presionar por sanciones a las industrias responsables de la contaminación de estos espa- 
cios de agua. Es ejemplo de ello el colectivo social denominado Asamblea y Territorio Sur para la recuperación de la cuenca del Tunjuelo-Fucha.

A partir de del año 2000 en Bogotá D. C. el agua se convirtió en un factor determinante en la planeación del territorio. En el Plan de Ordenamiento Territorial (POT) del año 2004, el Distrito comienza a reconocer la gestión integral del recurso hídrico por parte de la comunidad, apoyando a los ciudadanos que iniciaron la lucha ambiental en varias localidades, lográndose la recuperación de humedales como Córdoba, Santa María del Lago, El Burro y La Vaca.

Los expertos mundiales en la gestión del recurso hídrico han estado recomendando en los últimos años, una política pública de gobernanza del agua, concepto muy diferente al de gobernabilidad, que es cuando el Estado solo genera políticas internas y desconoce la gestión de actores sociales. El concepto de gobernanza del agua en Bogotá D. C. fue esencial, a partir del año 2012, para vincular las peticiones de la comunidad, expresadas a través de la instalación de los Pre- cabildos y Cabildos de Gestión del Agua (CGA) realizados en diferentes localidades de la ciudad capital, generando interesantes jornadas de participación ciudadana.

\section{PROBLEMA DE INVESTIGACIÓN}

Los procesos industriales, la urbanización sin planeación y la constante contaminación han motivado a la movilización social. La investigación planteada pretende establecer si el proceso de gobernanza del agua en Bogotá D. C. ha generado espacios efectivos de participación ciudadana para la gestión integral del recurso hídrico en sus territorios.

¿Hasta dónde ha sido viable la participación ciudadana en la gestión de los espacios de agua? y ¿Han sido realizables algunas peticiones de los CGA en Bogotá, para mejorar la gestión integral del recurso hídrico? son los interrogantes que la investigación pretende resolver.

\section{METODOLOGÍA}

Se trata de una investigación de tipo documental, que describe y analiza el proceso de gobernanza del agua que se ha venido desarrollando en Bogotá D. C., como elemento esencial para generar espacios de participación ciudadana en el manejo del recurso hídrico en sus territorios. La investigación se enmarca dentro del campo de la investigación socio-jurídica y socio-ambiental, estable- 
ciendo un diálogo documental a partir de las evidencias de estos procesos de participación ciudadana.

\section{RESULTADOS DE LA INVESTIGACIÓN}

\subsection{Desarrollo histórico de la gobernanza del agua}

El documento de posicionamiento del VI Foro Mundial del Agua «Hacia una buena Gobernanza para la gestión integrada de los Recursos Hídricos» (2012) citando a Rogers y Hall (2003) y al Programa de las Naciones Unidas para el Desarrollo, UNDP (2000), establece el siguiente concepto de gobernanza del agua: «La gobernanza del agua se refiere a la interacción de los sistemas políticos, sociales, económicos y administrativos que entran en juego para regular el desarrollo y gestión de los recursos hídricos y la provisión de servicios de agua a diferentes niveles de la sociedad». Este concepto ha venido evolucionando a través de los diversos Foros Mundiales del Agua que se han venido realizando a partir del año 2000, exigiéndose menos protagonismo del Estado y mayor intervención de la comunidad en la gestión del agua.

Este documento destaca la importancia de desarrollar la buena gobernanza del agua en varios países de América, generando un fortalecimiento en los actores sociales y un buen entendimiento con los gobiernos respectivos. La gobernanza se enmarca no como un fin, sino como un proceso permanente de participación ciudadana. La gobernanza se relaciona con un concepto dinámico, en el que intervienen actores gubernamentales y no gubernamentales.

El VI Foro Mundial del Agua, a través del documento mencionado, diferencia claramente el concepto de gobernanza y gobernabilidad. La gobernabilidad es un proceso o mecanismo de implementación de la administración, sin la intervención de los actores sociales; en cambio la gobernanza, concepto amplio, es un proceso en el cual opera la sociedad, en una modalidad democrática, que pueda generar un manejo del recurso hídrico con justicia social. El documento reitera el reto para América: consolidar la gobernabilidad de los recursos hídricos a través de procesos democráticos.

Ya se había planteado en el II Foro del Agua celebrado en Holanda, según lo relata el documento del VI Foro del Agua que se ha venido citando, que «la crisis del agua que se vive es una crisis de gobernanza, no sólo de gobernabilidad (OECD, 2001)». En el Documento Marco para la Acción de dicho Foro, se identificó a la GIRH como una herramienta clave para proporcionar seguridad hídrica al desarrollo de la humanidad. 
El documento referido aborda los tres problemas relacionados con la gobernanza del agua: 1) la administración del agua a partir de la unidad de ciclo hidrológico (aguas superficiales y aguas subterráneas) para gestionarse integralmente, 2) la provisión de servicios eficientes para hacer efectivo y universal el acceso al agua potable y saneamiento y 3) la formulación e implementación de políticas efectivas, transversales, coordinadas, especialmente con las territoriales y la acción subsidiaria de los gobiernos cuando sea posible. La experiencia en la región demuestra que si se construyen colectivamente las soluciones y se involucran más actores sociales en la toma de decisiones, hay más garantía de permanencia y continuidad de las instituciones (p. 8).

El planteamiento de la gobernanza del agua entró muy pronto en la controversia mundial sobre el manejo del agua. Los defensores del agua como un derecho sostuvieron que la gobernanza de esta no era más que un disfraz de la privatización del agua, para generar una mercancía o necesidad económica de ella, controlada por las multinacionales europeas. Los Foros del Agua han venido proponiendo la gobernanza del agua con justicia social, para reivindicar y garantizar el derecho humano al agua, reconocido en la Organización de las Naciones Unidas (ONU) en el año 2002, mediante la observación nro. 15 de los derechos Económicos, Sociales y Culturales (ONU, 2002).

La ONU sostiene que el proceso de gobernanza del agua se debe fortalecer no solamente con políticas públicas, sino con procesos democráticos de participación ciudadana. A propósito, el ambientalista colombiano Rafael Colmenares (2013) sostiene que

Si la «gobernanza del agua» significa, de veras, algo diferente a lo anterior, el concepto debería nutrirse de los principios de la «justicia del agua» (...) propuestos hace algún tiempo por Maude Barlow y parten del reconocimiento del derecho humano al agua.

Refiriéndose a Maude Barlow, señala Colmenares que fue ella como «activista y premio Nobel alternativo, presidenta del Consejo de los Canadienses, quien como asesora del presidente de la Asamblea de la ONU, en 2010, inspiró la resolución 64/292 del 28 de julio de aquel año, que reconoce aquel derecho». Esta resolución fue seguida, según Colmenares, por la importante resolución del 10 de septiembre del mismo año, del Consejo de Derechos Humanos de la ONU, según la cual «El derecho al agua y al saneamiento es un derecho humano, igual a todos los demás derechos humanos, lo que implica que es justiciable y de aplicación obligatoria».

Queda para la reflexión, la construcción teórica lograda hasta ahora por reconocidos estudiosos del tema de la gobernanza y la gobernabilidad del agua 
para una GIRH en Latinoamérica. Autores como Peter Rogers (2001), Solanos y Jouravlev (2001) y Domínguez (2010) han dejado claro que

La noción de gobernanza, cuando es aplicada al agua, se refiere a la capacidad de un sistema social para movilizar las sinergias de una forma coherente, para el desarrollo sustentable de los recursos hídricos. La noción incluye la habilidad de diseñar políticas públicas (y movilizar recursos sociales en apoyo de ellas), que son socialmente aceptadas y que tienen como meta el uso y desarrollo sustentable de los recursos hídricos, y lograr una implementación efectiva por los diferentes actores involucrados en el proceso. Así, en un primer momento, dentro de un enfoque de gobernanza del agua se busca coordinar las acciones de los diversos sectores y grupos de interés, a diferentes escalas, desde la local, regional, nacional, y hasta la internacional (VI Foro Mundial del Agua, 2012, p. 16).

El tema de la gobernanza del agua en los últimos años ha tomado mucha transcendencia para los países americanos, hasta el punto de hacer parte de las condiciones que exige la banca mundial y el Fondo Monetario Internacional (FMI), para efecto de préstamos: una buena gobernanza en la GIRH.

Es también de vital importancia la vinculación de los sectores socio-culturales en las decisiones acerca del recurso hídrico, en la perspectiva de evitar conflictos de la magnitud del presentado en la ciudad de Cochabamba, Bolivia, en el año 2000, conocido como «guerra del agua». El gobierno boliviano, sin tener en cuenta las problemáticas, intereses y expectativas de las comunidades de la ciudad de Cochabamba, emitió la Ley 2066, sobre Alcantarillado Sanitario, abriendo la puerta a la concesión de los servicios de abastecimiento de Agua Potable y Alcantarillado. Fue una adjudicación poco clara del contrato de Servicio Municipal de Agua Potable y Alcantarillado (Semapa) y la realización del proyecto múltiple Misicuni, asuntos que se expresaron para la población en un exagerado y arbitrario incremento de las tarifas y una pésima prestación de los servicios contratados. Fueron varias semanas de violentos enfrentamientos de la población con la fuerza pública; hubo varios muertos y cientos de detenidos. Finalmente, la empresa extranjera huyó del país y la mencionada Ley fue derogada (De La Fuente, 2000).

\subsection{Procesos de participación ciudadana en la gestión hídrica en Bogotá D.C.}

Tanto los marcos jurídicos constitucionales de 1991, como las leyes referidas a procesos de participación ciudadana, la Ley 134 de 1994 o Ley Estatuaria de los mecanismos de participación del pueblo, y la Ley 142 de 1994 o Ley de servicios públicos domiciliarios, junto a acciones constitucionales, facilitaron la conformación de actores sociales y el desarrollo de procesos de participación 
ciudadana para la gestión del recurso hídrico en Bogotá D. C. Procesos que en los últimos años fueron articulados con la política pública del Distrito y la gobernanza del agua, como gestión integral en el ciclo del agua.

La reciente historia de la participación ciudadana para la gestión del recurso hídrico en Bogotá D. C. evidencia dos importantes procesos, en algunos casos simultáneos; en otros se entrecruzan. Ellos son: 1) participación ciudadana en la recuperación de cuencas y humedales; y 2) conformación de Cabildos del Agua.

\subsubsection{Participación ciudadana en recuperación de cuencas y humedales}

Como respuesta al dramático deterioro de humedales y cuencas hidrográficas en la ciudad de Bogotá D. C., en los años noventa, importantes sectores de la población se organizaron en amplios movimientos sociales participativos, con el propósito de recuperar esos importantes ecosistemas, especialmente en las localidades del Sur, para mitigar los efectos del deterioro ambiental en las cuencas de los ríos Fucha, Tunjuelo y Bogotá.

Para ese entonces los humedales, ecosistemas de aguas y de biodiversidad, padecían de la siguiente problemática: baja valoración social, degradación ecológica, desecación para agricultura y ganadería, rellenos para urbanización, contaminación por vertimiento de aguas residuales y afectación de la salud de la comunidad aledaña, fragmentación por proyectos viales, etc. (Fundación Humedal La Conejera, 1993).

Humedal La Conejera. En 1993, habitantes de los barrios localizados en la localidad de Suba, con el liderazgo del señor Germán Galindo Hernández, conformaron un Comité Ecológico que más tarde se constituyó en Fundación Humedal la Conejera, con el propósito de recuperar este ecosistema de más de 60 hectáreas que se encontraba en vía de extinción. Hasta ese entonces el humedal había recibido más de 500 volqueadas de escombros y basura de los barrios cercanos, afectando cientos de especies de flora y fauna nativas (Nullvalue, 1998).

La iniciativa de la comunidad en su manejo de gobernanza del espacio de agua creó la fundación que durante más de 20 años ha velado por la conservación de este ecosistema. La comunidad ha ejercido una vigilancia de 24 horas durante todos estos años, para impedir el vertimiento de escombros de volquetas de los nuevos proyectos de vivienda en la localidad de Suba; se organizan permanentemente brigadas de limpieza, cabildos locales de pedagogía ambiental, y la recuperación y construcción de más de 220 senderos (Nullvalue, 1998). 
La siguiente es la problemática identificada por la Fundación Humedal La Conejera: 1) rellenos y urbanización ilegal; 2) desaparecido el $50 \%$ del humedal; 3) vertimiento de 5 millones de litros diarios de aguas residuales; 4) actividades agrícolas y ganaderas dentro del cauce y área de protección adyacente; 5) pérdida total del bosque protector; 6) cacería incontrolada de aves y curíes; 7 ) presencia de manadas de perros asilvestrados depredadores de la fauna, y 8) indiferencia total del Estado y la ciudadanía (Fundación Humedal La Conejera, 1993).

Un fallo de tutela interpuesta por Germán Galindo obligó a la Empresa de Acueducto y Alcantarillado de Bogotá (EAAB) y a otras entidades estatales a descontaminar el humedal y además construir la infraestructura necesaria para el desagüe de las aguas residuales. Hoy el humedal recibe más de 16.000 visitantes al año, complacidos ante el maravilloso espectáculo que conforma la vegetación nativa de los humedales, la fauna silvestre y los patos canadienses que llegan cada año a visitar los espejos de los humedales.

Humedal de Córdoba. Ubicado entre la avenida Boyacá y avenida Córdoba, jurisdicción de las urbanizaciones de Niza, en Bogotá. La comunidad, desde 1998, ha gobernado este espacio de agua, liderada por ciudadanos activistas como Mauricio Castaño, Luz Marina Gómez, Luis Jorge Vargas, Liborio Sánchez y Myriam Luján, quienes se han opuesto a la construcción de una ciclovía y de una plazoleta, lo cual implicaría talar árboles, destruir el ecosistema. El mismo Departamento Administrativo de Medio Ambiente (DAMA), en ese entonces, sostenía que esas construcciones no afectarían el ecosistema.

A pesar de la oposición de una parte de la comunidad, Mauricio Castaño y sus compañeros ambientalistas continuaron en la costosa empresa, incluso con recursos de sus propios bolsillos, para la recuperación del ecosistema y sembrar más de 3500 árboles. Finalmente, se hizo justicia en el año 2001 al ganar una acción popular y se logró que la Corte Constitucional sentenciara que el Acueducto de Bogotá no podría ejecutar ningún tipo de proyecto en el humedal si no se contaba con el aval de la comunidad (Escobar, 2013).

Humedal El burro. Localizado en la localidad de Kennedy, viene siendo afectado desde hace varios años por los vertimientos de aguas residuales, desechos industriales y la presencia de población indigente. Se ha buscado recuperar ese espacio de agua a través de la comunidad, liderada por el edil de la localidad de Kennedy, Alejandro Torres, y mediante la gestión de la ONG Humedales de Bogotá, junto a grupos de jóvenes ambientalistas empeñados en recuperar los humedales de Bogotá D. C., bajo la dirección de Jorge Manuel Escobar. 
Dicha ONG, a través de gestiones ambientales realizadas por los propios ciudadanos, ha impedido desde hace 15 años, la construcción de proyectos urbanísticos que acabarían con el humedal. También se ha logrado implementar la Escuela Juvenil de Ecología Política. Además, la comunidad organizada en estos grupos ambientales también está trabajando por la recuperación del humedal El Burrito, un antiguo brazo del humedal El Burro, donde aún sobreviven ranas y biodiversidad propia de humedales.

La comunidad de la localidad de Kennedy también libró una lucha en contra de las constructoras que pretendían, a principios del año 2012, el encerramiento de la Cola del Burro, como lo denominaron sus defensores, a un ecosistema olvidado por las autoridades ambientales. La lucha dio magníficos resultados; la resolución 1238 de 2012 de la Secretaría de Medio Ambiente reconoció esta área como área de protección ambiental.

La cuenca del Tunjuelo. El río Tunjuelo atraviesa barrios desde Usme hasta Soacha, siendo afectado por graves problemas ambientales generados por la minería, las curtiembres de San Benito, la cementeras Cemex, los rellenos sanitarios, las invasiones de la ronda del río y la expansión urbana. Además, las cementeras Cemex y Holcim y la fundación San Antonio desviaron el cauce del Tunjuelo y afectaron su calidad hídrica.

La comunidad de estos barrios se movilizó en el año 2006, realizando un Cabildo para exigir a las autoridades distritales que impidieran la desviación del cauce del río Tunjuelo. De igual manera, se logró integrar una mesa de trabajo, con la participación de algunos movimientos urbanos como Territorio Asamblea Sur y la Red Juvenil de Territorio Sur.

La organización de Territorio Sur se originó en el año 1998, con el propósito de desarrollar un proceso de educación ambiental y ancestral, con los jóvenes de la localidad sexta de Bogotá. El proceso formativo se ha venido desarrollando a través de la Escuela de Liderazgo del Territorio Sur y en la participación de foros ambientales y comunitarios. En el 2003, ser realizó el Primer Foro Regional de Jóvenes y Territorio Sur, en el marco de los conflictos ambientales de la cuenca, en el que participaron alrededor de 500 jóvenes; el evento se realizó en el Instituto Nacional de Educación Media (INEM) del Tunal. En el 2004, organizaron el Segundo Foro Regional, teniendo como temas: el agua y sus conflictos por uso y manejo, y los programas de conservación y ordenamiento de la cuenca (García y Ramírez, 2008 ).

Estos foros ambientales hicieron posible el surgimiento de más organizaciones, entre ellas la corporación Casa Asdoas, en Usme, que recoge la memoria histórica, ancestral, cultural y ambiental de la localidad; en la localidad de San 
Cristóbal aparece la organización Paraíso Colombia; y en la localidad de Kennedy, la Red Juvenil Territorio Sur que integra a estudiantes de varios colegios para desarrollar con ellos, procesos de participación juvenil.

Otro proceso de participación ciudadana fue Asamblea Sur, que tiene su comienzo en 1993 por iniciativa de líderes de Territorio Sur, al integrar un comité que tenía como propósito el reconocimiento del parque Entre-Nubes y el manejo por parte de la comunidad de la cuenca del Tunjuelo. Fueron los estragos de la inundación de río los que motivaron a la instalación de la Asamblea Sur en la que participaron importantes organizaciones sociales como el Sindicato de Trabajadores de la ETB, el Sindicato de Trabajadores de la Universidad Distrital y el movimiento agrario de Usme y Sumapaz, y a raíz de los estragos de inundación del río se integró Asamblea Sur, líderes comunales y redes de jóvenes ambientalistas del sur.

La Asamblea Sur llegó a plantear en su momento «que se reconozca a la Cuenca del río Tunjuelo como un territorio único, que comparte un proceso histórico de constitución y que como tal debe reconocerse en las propuestas de intervención y manejo» (Asamblea Sur, 2006). Bajo el lema «Somos Urbano-Rural: de Sumapaz a Bosa» se realizaron acciones de difusión en la cuenca para posicionarse como actores válidos en la comunidad (García y Ramírez, 2008).

La Asamblea Sur tiene identificada, desde el año 2006, la grave problemática ambiental que afecta la cuenca del río Tunjuelo $(73 \mathrm{~km}$ de cauce que atraviesa varias localidades de Bogotá D. C.):

En la cuenca existen en medio de los barrios alrededor de 450 industrias peleteras que contaminan el río; el relleno sanitario Doña Juana, que sufrió un grave deslizamiento de basuras y lixiviados que puso en riesgo la salud de la población del sector y de toda la Capital y aumentó la contaminación del río; y los parques mineros, cuya explotación de gravas, gravillas y arenas en las vegas del río, para producir mezclas de concreto, ha dejado en la zona extensas excavaciones, algunas de ellas de más de 80 metros de profundidad, con derrumbes frecuentes y retención de las aguas lluvias que se han constituido en focos de plagas y contaminación (Coordinación Asamblea Sur, 2006).

Frente a las inundaciones en la parte baja del rio Tunjuelo, la Asamblea Sur planteó:

La inundación del barrio Tunjuelito, el desvío del cauce del río y el estancamiento de 30 millones de $\mathrm{m}^{3}$ de agua contaminada en Las cárcavas de las minas entre 
la Vía a Villavicencio y la Av. Caracas, y los barrios Aurora al sur y México, Tunjuelito y San Benito al norte, amenaza con una catástrofe urbana que de causarse sería peor que la desaparición de Armero (Tolima). (Peña, 2008).

\subsubsection{Procesos de conformación de Cabildos del Agua}

El año 2012 marca el inicio de los procesos de participación de las comunidades en la gobernanza del agua, con la conformación de los 43 Cabildos en todas las localidades de Bogotá, denominados Cabildos Gobernanza del Agua (CGA). La implementación de estos Cabildos integró las cuencas y las microcuencas del Distrito a fin de incorporar a la comunidad y el tejido social en la recuperación de los espacios de agua.

Una primera estrategia para lograr estos propósitos fue la de implementar los Precabildos del Agua, en coordinación con el equipo interinstitucional liderado por la Secretaría Distrital de Ambiente y la participación de la Secretaría del Hábitat, la Unidad Administrativa Especial de Servicios Públicos (UAESP), el Fondo para la Atención de Emergencias (Fopae), y la Empresa de Acueducto y Alcantarillado de Bogotá (EAAB), entre otras entidades.

Es de destacar el Precabildo del río Tunjuelo, al cual asistieron las localidades de Sumapaz, Usme, Kennedy, Uribe Uribe, Bosa, San Cristóbal y Ciudad Bolívar. Los temas previstos que se agotaron en las mesas de trabajo fueron: los ecosistemas, páramo y ruralidad, vertimientos, escombros y residuos, minería, deslizamientos, inundaciones, ordenamiento, servicios ambientales, rondas hídricas e hidráulicas y zonas inundables, memoria ancestral del territorio, regalías y responsabilidad social y compensación. También se destacó el Precabildo de la Localidad de Puente Aranda, con actividades de sensibilización y activación ciudadana para proteger el río Fucha y sus humedales aledaños.

Posteriormente se desarrollaron 34 CGA en varias de las localidades de Bogotá D. C. En el caso de la localidad de Puente Aranda, bajo el nombre de Cabildo Participativo de Gobernanza del Agua, se generaron las siguientes estrategias en los temas ambientales:

- Educación ambiental, manejo de residuos, cambio climático, estructura ecológica principal, flora y fauna, espacio del agua.

- Programas dirigidos a la comunidad en saneamiento ambiental y salud ambiental.

- Educación ambiental (en temas relacionados con manejo de residuos, residuos peligrosos y electrónicos, contaminación electromagnética, código de policía, tec- 
nologías limpias, cambio climático, ciencias tecnológicas y/o ambientales) dirigida a la comunidad, colegios, policía ambiental y empresas con el compromiso de replicar la información en la comunidad.

- Crear un Centro de Información y Educación Ambiental local.

- Creación de la empresa social de reciclaje en la localidad de Puente Aranda. Actualización del mapa local de riesgos químicos en la localidad.

- Promoción de brigadas comunitarias en gestión integral del riesgo, viviendas en amenaza de ruina y otros factores de riesgo (Cabildo Participativo de Gobernanza del Agua en Puente Aranda Humana, Moderna e Industrial «decisión final», 2012).

Los CGA en Bosa se concentraron en varias estrategias territoriales de participación ciudadana en temas como: río Tunjuelo, humedales Tibanica y La Isla, quebrada Tibanica, canales de aguas lluvias y río Bogotá. En el informe se destacan las siguientes actividades y resultados en los recorridos interpretativos con gran participación ciudadana (Cabildos Gobernanza del Agua Bosa, 2012):

\section{Recorridos interpretativos}

\section{Territorio Problema identificado}

Río Tunjuelo - Deficiente cultura Ambiental.

- Inadecuada Disposición de Escombros y ordinarios.

- Necesidad de declarar el predio la Isla como Humedal.

- Ocupación ilegal de la ZMPA.

- Necesidad de vincular jóvenes a los procesos de presupuestos participativos.

Humedal Tibanica • Inadecuada cultura ambiental.

- Vulnerabilidad del ecosistema al no existir un cerramiento total del humedal y una administración constante.

- Inadecuada disposición de escombros y residuos ordinarios.

Río Bogotá - Inadecuada cultura ambiental.

- Deficiente gestión de la EAAB en la limpieza de canales.

- Las comunidades no se encuentran preparadas en la gestión del riesgo.

Fuente: Cabildos Gobernanza del Agua localidad de Bosa, octubre de 2012.

En los cinco CGA en Bosa, la comunidad presentó más de 48 peticiones sobre temas tales como: sensibilización en basura cero y gobernanza del agua, incen- 
tivos económicos, control ambiental en los espacios del agua y construcción de aulas ambientales. Este Cabildo logró integrar, desde el año 2012, un comité social conformado por 85 ciudadanos de las respectivas UPZ de la localidad; se logró también una mayor conciencia acerca de la importancia de los espacios de agua; también se logró la siembra de árboles en la ronda del río Tunjuelo, con la participación de más de 31 barrios en la localidad (Cabildos Gobernanza del Agua, localidad de Bosa, 2012).

En la localidad de Teusaquillo, con el nombre de Cabildos de Presupuesto Participativo de la Gobernanza del Agua, se priorizaron las siguientes iniciativas Medio Ambiente (Secretaría Distrital de Ambiente, 2013):

- Ordenamiento ambiental del territorio con participación vinculante de la comunidad teniendo en cuenta la renaturalización de las cuencas de la localidad y el proyecto «Recuperación ambiental y paisajística de la zona de ronda del río Arzobispo».

- Demoler y/o tumbar el muro que oculta el canal del río Arzobispo, en la carrera 30 con calle 49, al lado del edificio de la lotería de Cundinamarca.

- Construcción de un puente peatonal sobre el canal del río Arzobispo, en la carrera $28^{\text {a }}$ con calle 49, al lado de la Casa de la Madre y el Niño.

- Arborizar y recuperar el corredor natural del borde norte del canal del río San Francisco, desde la carrera 50 hasta la carrera 68.

Con los CGA desarrollados en las localidades de Bogotá, desde el año 2012, se dio inicio a los estudios de recuperación de espacios de agua, a la identificación de cuencas y microcuencas en el Distrito y a la generación de la cultura de gobernanza del agua en la comunidad. Para efecto de los estudios señalados, los diagnósticos pertinentes deberían estar dirigidos hacia las siguientes variables (Secretaría Distrital de Ambiente, 2013):

- Principales cuencas del distrito (Torca-Guayarla, Salitre, Fucha y Tunjuelo).

- Territorios ambientales (Borde Norte, Cerros Orientales, Salitre, Fucha, Tunjuelo, Humedales, Río Bogotá y Páramo-Ruralidad).

En estos 43 CGA se presentaron más de 300 peticiones, priorizadas en las siguientes categorías, según información suministrada por la Secretaría de Medio Ambiente (2013):

- Contaminación 
- Espacios del agua

- Ética y educación ambiental

- Gestión ambiental institucional

- Gestión ambiental comunitaria

- Ordenamiento ambiental del territorio

- Estructura ecológica principal

- Gestión del riesgo

- Fauna

- Minería

Iniciativas priorizadas en los CGA. La política del Distrito, desarrollada a través de los Cabildos de Gobernanza del Agua (CGA) tiene como propósito la implementación del Plan de Desarrollo Distrital «Bogotá Humana» contemplado en los programas de Gobernanza del agua, Gestión del riesgo y Basura cero; para ello ha sido necesario el fortalecimiento de la apropiación del territorio por parte de las comunidades asentadas en ellos, promoviendo la participación efectiva y decisoria y el diálogo. La comunidad, a través de los 43 CGA ha presentado varias peticiones, las cuales han venido siendo atendidas por las entidades distritales como el Jardín Botánico, la Secretaría de Medio Ambiente y la EAAB, entre otras. Ver tabla página siguiente.

La Secretaría Distrital de Ambiente procedió a realizar un seguimiento al cumplimiento de estas peticiones, a través de los Comités de Control Social, descartándose las peticiones no viables y finalmente priorizando 288 iniciativas en las 20 localidades. También se ha tramitado una metodología para la implementación de talleres, la coordinación de acciones y la articulación de recursos para responder a las iniciativas priorizadas. Como resultado de los talleres se generaron acciones conjuntas teniendo en cuenta que las competencias jurídicas y administrativas requieren la participación de los distintos niveles de la administración.

El derecho a la participación ciudadana en las localidades de Bogotá a través de los CGA ha representado no solamente la defensa del agua como derecho, sino además la posibilidad de avanzar frente a la problemática social y el deterioro ambiental en Bogotá. No todo ha sido intervención directa del Estado, 


\begin{tabular}{|c|c|c|}
\hline Localidad & Iniciativa CGA & Categoría \\
\hline \multirow{3}{*}{ Usaquén } & $\begin{array}{l}\text { Procesos de educación: Ser más pedagógicos y cercanos con la } \\
\text { comunidad. }\end{array}$ & $\begin{array}{l}\text { Ética y Educación } \\
\text { Ambiental }\end{array}$ \\
\hline & Acueducto y alcantarillado: Intervenciones de la EAAB. & Espacios del Agua \\
\hline & Basura cero: Campañas y gestión ambiental de entidades. & \\
\hline \multirow{3}{*}{ Chapinero } & $\begin{array}{l}\text { Jornadas prácticas: organización comunitaria, gestión del riesgo y } \\
\text { manejo de residuos. }\end{array}$ & $\begin{array}{l}\text { Gobernanza Ambiental } \\
\text { Comunitaria }\end{array}$ \\
\hline & $\begin{array}{l}\text { Recuperación de quebradas (saneamiento en las quebradas Delicias y el } \\
\text { Chulo). }\end{array}$ & $\begin{array}{l}\text { Estructura ecológica } \\
\text { principal }\end{array}$ \\
\hline & Recuperación de nacimientos de agua. & \\
\hline Santa Fe & $\begin{array}{l}\text { Crear el corredor ecológico de rondas, que contemple espacios verdes en } \\
\text { los barrios, que tengan un uso ambiental hacia la comunidad, en el } \\
\text { sentido de recreación, formación y acción. }\end{array}$ & $\begin{array}{l}\text { Estructura Ecológica } \\
\text { Principal }\end{array}$ \\
\hline $\begin{array}{c}\text { San } \\
\text { Cristóbal }\end{array}$ & $\begin{array}{l}\text { Control de especies invasoras y proceso de restauración ecológica: } \\
\text { Quebradas Chorro Colorado, Chiguaza, la Nutria, Berejones y Morales; } \\
\text { Cerros Orientales. }\end{array}$ & $\begin{array}{l}\text { Estructura Ecológica } \\
\text { Principal }\end{array}$ \\
\hline \multirow{7}{*}{ Usme } & $\begin{array}{l}\text { Construcción de colectores y sistemas de alcantarillado en barrios altos } \\
\text { (Tocaimita, La Flora, Sucre III, Uval I). }\end{array}$ & \multirow{7}{*}{ Espacios de Agua } \\
\hline & $\begin{array}{l}\text { En el barrio Villa Hermosa canalización de aguas lluvias en el contorno } \\
\text { de la loma de la calle } 94 \text { a la diagonal 97A y entre la carrera 7C y la 7F } \\
\text { para evitar problemas de inundación de viviendas. }\end{array}$ & \\
\hline & Destapar alcantarillado entre la calle 94 y la 98 , entre la carrera 6 y la $7 \mathrm{~F}$. & \\
\hline & $\begin{array}{l}\text { Limpieza y mantenimiento de cuerpos de agua, a través de la } \\
\text { promoción de empleo local. }\end{array}$ & \\
\hline & $\begin{array}{l}\text { Mantenimiento integral Quebrada Chuniza, Yomasa, El Piojo, Medianía } \\
\text { y Arrayanal, identificando riesgo, fauna y flora, cartografía social y } \\
\text { seguimiento. }\end{array}$ & \\
\hline & $\begin{array}{l}\text { Plan de Ordenamiento de la Cuenca Tunjuelo (desde Chuniza hasta la } \\
\text { parte alta). }\end{array}$ & \\
\hline & Construcción de Alamedas. & \\
\hline \multirow{2}{*}{ Tunjuelito } & Dragado del río Tunjuelo. & \multirow{2}{*}{ Espacios de agua } \\
\hline & Senderos ecológicos en el humedal. & \\
\hline \multirow{2}{*}{ Bosa } & Control y vigilancia a toda la ronda del río Tunjuelo. & \multirow{2}{*}{$\begin{array}{l}\text { Estructura ecológica } \\
\text { principal }\end{array}$} \\
\hline & Preservación del pulmón de Bavaria. & \\
\hline \multirow[b]{2}{*}{ Usme } & Plan de ordenamiento de la cuenca del Tunjuelo y de sus quebradas. & \multirow[b]{2}{*}{ Espacios de agua } \\
\hline & $\begin{array}{l}\text { Construcción de gaviones en la parte alta de la loma para canalización } \\
\text { de agua-lluvias. }\end{array}$ & \\
\hline Fontibón & Restauración ecológica y gestión del riesgo. & Espacios de Agua \\
\hline \multirow{2}{*}{ Kennedy } & Sensibilización y educación ambiental a las comunidades. & \multirow[t]{2}{*}{ Espacios del Agua } \\
\hline & Revisar el colector de aguas ${ }^{1}$ & \\
\hline Engativá & $\begin{array}{l}\text { Conformación e implementación de una escuela ambiental, la cual } \\
\text { tendría la responsabilidad de liderar procesos articulados: proyecto } \\
\text { basura cero desde la fuente, gobernanza del agua, gestión del riesgo, } \\
\text { cuidado y tenencia de mascotas, talleres de agricultura urbana y } \\
\text { reforestación con especies nativas, compostaje, manual de silvicultura } \\
\text { urbana, responsabilidad ciudadana en el cuidado y protección del } \\
\text { Medio Ambiente. }\end{array}$ & $\begin{array}{l}\text { Ética y Educación } \\
\text { Ambiental }\end{array}$ \\
\hline
\end{tabular}

Fuente: Secretaria Distrital de Ambiente (2014). Iniciativas Cabildos Gobernanza del Agua. 
ha sido más bien una estrategia para gobernar los espacios de agua por parte de la sociedad civil, como en los años 90 .

Los CGA en las localidades de Bogotá han generado procesos de participación ciudadana en atención a las necesidades prioritarias, a través de instrumentos como la educación y la pedagogía ambiental. La comunidad en muchas ocasiones ha solicitado la atención de muchas peticiones, que durante años la administración distrital no atendió; algunas han tenido respuestas, pero otras solicitudes no se han implementado a pesar de haberse desarrollado un seguimiento por parte de los funcionarios estatales encargados de ello.

Con estos procesos de participación ciudadana en las localidades de Bogotá, promovidos por la administración distrital, desde el 2012, se ha consolidado un acercamiento más directo con los procesos populares de gestión ambiental que tienen antecedentes históricos muy importantes en los sectores de Bogotá más deprimidos y afectados por el deterioro de los ecosistemas.

\section{CONCLUSIONES Y RECOMENDACIONES}

El debate mundial sobre la problemática ambiental hace evidente que existe una notable diferencia entre los conceptos de gobernanza y gobernabilidad del agua; el primero se refiere a la interacción de los actores sociales con actores públicos y privados en la gestión del recurso hídrico, con el fin de concertar estrategias y acciones ciudadanas; el otro concepto es, en muchos casos, una imposición de políticas públicas en la gestión de los recursos hídricos, con desconocimiento de los intereses y expectativas de las comunidades asentadas en esos territorios.

Como respuesta a la negligencia del Estado y a la carencia de una política pública ambiental en Bogotá, desde los años noventa se ha venido generando un empoderamiento de la comunidad, de los sectores sociales afectados, en términos de conocimiento técnico científico y una participación ciudadana, incidiendo en la formulación y ejecución de las políticas públicas ambientales.

A través del ejercicio de acciones constitucionales, como las acciones de tutela y las acciones populares, se han logrado fallos importantes que han beneficiado a la comunidad, exigiéndole al Estado, entre otras, mejorar los servicios públicos y recuperar las quebradas de la contaminación ambiental.

Los CGA han generado procesos democráticos de participación ciudadana y han vinculado a las comunidades a la conservación de los ecosistemas y a la administración de los espacios de agua, para mejorar las condiciones de vida. 
Los CGA realizados en las localidades de Bogotá a partir del año 2012 han permitido recoger las iniciativas populares frente a la problemática ambiental y establecer un orden de prioridades de las mismas. Algunas peticiones no han logrado concretarse en su tratamiento, debido a factores como la falta de información o la presencia débil de las comunidades en algunas localidades.

Las comunidades han venido desarrollando interesantes procesos de educación y pedagogía ambiental, lo mismo que la creación de los vigías hídricos, la convocatoria de mingas y talleres y recorridos de interpretación en sus espacios de agua. Esto se traduce en el reconocimiento y apropiación del derecho colectivo a gobernar sus propias fuentes hídricas.

La investigación encontró que los CGA tienen como sus antecedentes históricos, los movimientos sociales y cívicos que se originaron en los años noventa, en la defensa de sus territorios, deteriorados por la problemática ambiental, la expansión urbana, la invasión de la ronda de los ríos y el mal manejo de los desechos industriales y tóxicos. Sin embargo, hay que señalar que las experiencias de los movimientos sociales y la instalación y desarrollo de 43 CGA no han sido suficientes en sus alcances, para un goce pleno en la gestión del recurso hídrico, lo que permite concluir que una buena gobernanza del agua se debe fundamentar principalmente en el liderazgo de las comunidades y en la búsqueda del consenso de todos los actores intervinientes, lo cual permitirá generar políticas públicas con justicia social en la gestión del agua.

\section{REFERENCIAS}

Cabildos Gobernanza del Agua Localidad de Bosa. (2012). Priorización de acciones. Recuperado el 25 de noviembre de 2014, de http:/ / oab.ambientebogota.gov.co/ es/el-observatorio-y-las-localidades/documentos-bosa/informe-cabildosgobernanza-del-agua-en-la-localidad-de-bosa

Cabildo Participativo de Gobernanza del Agua en Puente Aranda Humana, Moderna e Industrial «decisión final». (2012). Recuperado el 25 de noviembre de 2014, de http://www.bogotasocial.org/las-localidades-hablan/puente-aranda/707-cabildo-participativo-de-gobernanza-del-agua-en-puente-aranda-humana-moderna-eindustrial-decision-final-

Colmenares, Rafael. (2013). ¿Gobernanza del agua o justicia del agua? Recuperado el 10 de octubre de 2014, de http:/ / viva.org.co/cajavirtual/svc0342/articulo12.html

Coordinación Asamblea Sur. (2004). La participación social en la solución de los conflictos del sur de Bogotá. Cuenca del río Tunjuelo. Recuperado el 10 de diciembre de 2014, de http:/ / es.slideshare.net/tigreblanco12/ ponencia-asamblea-sur 
De La Fuente, Manuel. (2000). La «Guerra» por el agua en Cochabamba. Crónica de una dolorosa victoria. Recuperado el 12 de noviembre de 2014, de http:/ / www.umss.edu.bo/Academia/Centros/Ceplag/AguaMDLF.PDF

Escobar, Mariana. (2013). Humedales tienen salvación. En: El Espectador, 1 de febrero de 2013. Recuperado el 20 de noviembre de 2014, de http:/ / www.elespectador.com/ noticias/actualidad/vivir/humedales-tienen-salvacion-articulo-402469

Fundación Humedal La Conejera. (1993). Recuperado el 15 de enero de 2015, de http: / / docplayer.es/14212740-Fundacion-humedal-la-conejera.html

García, Diego y Ramírez, Sandra. (2010). Procesos participativos en la cuenca río Tunjuelo. Recuperado el 1 de noviembre de 2014, de https:/ / app.assembla.com/ spaces/cdr/wiki/Procesos_Participativos_en_la_Cuenca_Rio_Tunjuelo

Nullvalue. (1998). Sobrevivientes de La Conejera. En: El Tiempo, 25 de enero de 1998. Recuperado el 15 de octubre de 2014, de http:/ / www.eltiempo.com/archivo/ documento/MAM-764931

ONU. (2002). 29. ${ }^{\circ}$ período de sesiones. Observación general n. ${ }^{\circ} 15$. Recuperado el 15 de octubre de 2014, de http:/ / www.solidaritat.ub.edu/observatori/general/ docugral/ONU_comentariogeneralagua.pdf

Peña, Flor Marina. (2008). Movimientos urbanos territoriales en la cuenca del río Tunjuelo en el sur de Bogotá (1980-2007). Bogotá D. C.: Universidad Distrital Francisco José de Caldas.

Secretaría Distrital de Ambiente (2014). Iniciativas Cabildos Gobernanza del Agua.

Secretaría Distrital de Ambiente. (2013). Avance iniciativas priorizadas Cabildos de Gobernanza del Agua Teusaquillo. Recuperado el 25 de noviembre de 2014, de http:/ / www.teusaquillo.gov.co/index.php/desarrollo-local/2013-09-23-23-42$27 /$ cabildos

VI Foro Mundial del Agua. (2012). Hacia una buena gobernanza para la gestión integrada de los Recursos Hídricos. Recuperado el 20 de octubre de 2014, de http:/ / www.gwp.org/Global/GWP-CAm_Files/Gobernanza\%20para\%20GIRH\% 202012.pdf 